\title{
CYP3A4 Mediates Oxidative Metabolism of the Synthetic Cannabinoid AKB-48
}

\author{
Niels Bjerre Holm, ${ }^{1,2}$ Line Marie Nielsen, ${ }^{1}$ and Kristian Linnet ${ }^{1}$
}

Received 14 April 2015; accepted 8 May 2015; published online 23 May 2015

\begin{abstract}
Synthetic cannabinoid designer drugs have emerged as drugs of abuse during the last decade, and acute intoxication cases are documented in the scientific literature. Synthetic cannabinoids are extensively metabolized, but our knowledge of the involved enzymes is limited. Here, we investigated the metabolism of $N$-(1-adamantyl)-1-pentyl-1H-indazole-3-carboxamide (AKB-48), a compound identified in herbal blends from 2012 and onwards. We screened for metabolite formation using a panel of nine recombinant cytochrome P450 (CYP) enzymes (CYP1A2, 2B6, 2C8, 2C9, 2C18, 2C19, 2D6, 2E1, and $3 \mathrm{~A} 4)$ and compared the formed metabolites to human liver microsomal (HLM) incubations with specific inhibitors against CYP2D6, 2C19, and 3A4, respectively. The data reported here demonstrate CYP3A4 to be the major CYP enzyme responsible for the oxidative metabolism of AKB-48, preferentially performing the oxidation on the adamantyl moiety. Genetic polymorphisms are likely not important with regard to toxicity given the major involvement of CYP3A4. Adverse drug-drug interactions (DDIs) could potentially occur in cases with co-intake of strong CYP3A4 inhibitors, e.g., HIV antivirals and azole antifungal agents.
\end{abstract}

KEY WORDS: adamantyl; cytochrome P450; metabolism; metabolites; synthetic cannabinoids.

\section{INTRODUCTION}

Synthetic cannabinoid designer drugs have emerged as drugs of abuse during the last decade and produce psychoactive effects resembling those of delta-9-tetrahydrocannabinol $\left(\Delta^{9}\right.$ THC), the main active constituent of cannabis, through binding to the same cannabinoid receptors $\mathrm{CB}_{1}$ and $\mathrm{CB}_{2}$ (1). The majority of compounds are chemically unrelated to $\Delta^{9}$-THC and are added to herbal material (known as "spice") before they are sold to the end-consumer via the Internet or through local head shops (2-4). According to the European Monitoring Centre for Drugs and Drug Addiction (EMCDDA), 30 new synthetic

Electronic supplementary material The online version of this article (doi:10.1208/s12248-015-9788-7) contains supplementary material, which is available to authorized users.

${ }^{1}$ Section of Forensic Chemistry, Department of Forensic Medicine, Faculty of Health and Medical Sciences, University of Copenhagen, Frederik V's Vej 11, 3rd floor, 2100, Copenhagen, Denmark.

${ }^{2}$ To whom correspondence should be addressed. (e-mail: niels.holm@sund.ku.dk)

ABBREVIATIONS: AKB-48, $N$-(1-Adamantyl)-1-pentyl- $1 H$ indazole-3-carboxamide; CES1, Carboxylesterase 1; CYP, Cytochrome P450; DDP-4, Dipeptidyl peptidase-4; DEA, Drug Enforcement Administration; DDI, Drug-drug interaction; EIC, Extracted ion chromatogram; EMCDDA, European Monitoring Centre for Drugs and Drug Addiction; FDA, Food and Drug Administration; HLM, Human liver microsomes; HR, High-resolution; IS, Internal standard; LC-MS, Liquid chromatography mass spectrometry; NPS, New psychoactive substance; RCYP, Recombinant CYP; THC, $\Delta^{9}$ Tetrahydrocannabinol cannabinoids were identified in 2014 bringing the total number to 134, thus making it the largest group of new psychoactive substances (NPS) within the EU (5).

Motivations for taking synthetic cannabinoids as opposed to cannabis can include curiosity, desire to experience another type of "high," availability, and to circumvent legislation and avoid detection following intake, e.g., in a forensic, psychiatric, prison, or workplace drug testing setting (6). The latter is possible, since immunoscreening assays rarely detect NPS. Liquid chromatography mass spectrometry (LC-MS) is commonly used to detect designer drugs $(7,8)$, but even though new compounds can be added to accurate-mass libraries solely based on exact masses, final confirmation and quantification require reference standards. The situation is complicated further when using urine as the sample matrix. Here, in addition, knowledge of the metabolic profile is essential as the synthetic cannabinoid parent compounds are usually not found in this matrix $(9,10)$. The biotransformations consist mainly of oxidation reactions to form hydroxylated and/or carboxylated metabolites, which are subsequently conjugated by UDP-glucuronosyltransferases (11).

Clinical symptoms of acute intoxication include tachycardia, slurred speech, nausea, and vomiting, while psychoactive symptoms include, e.g., dizziness, agitation, drowsiness, and anxiety $(6,12,13)$. Knowledge of the specific metabolizing enzymes will improve our ability to predict potential drugdrug interactions (DDIs) associated with increased risk of intoxication and possible impacts of genetic polymorphisms, but so far, the investigations of phase I metabolism are limited. For the JWH-type synthetic cannabinoids JWH-018 and AM-2201 (Fig. 1a), Chimalakonda et al. identified CYP1A2 and 2C9 as the major cytochrome P450 (CYP) 


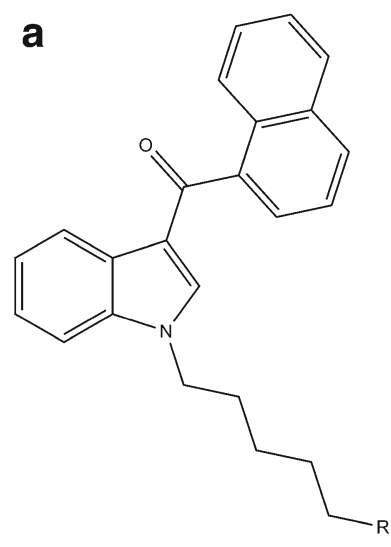

b

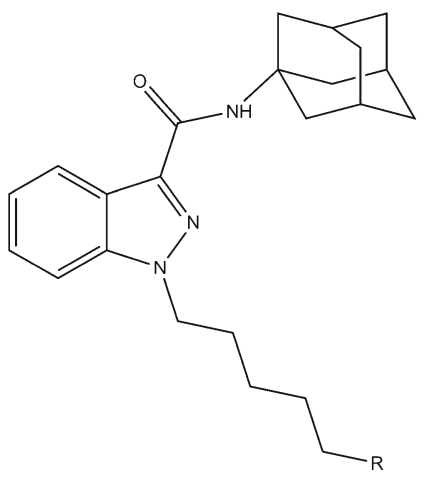

$$
\begin{aligned}
& \mathrm{R}=\mathrm{H}: \quad \mathrm{JWH}-018 \\
& \mathrm{R}=\mathrm{F}: \mathrm{AM}-2201
\end{aligned}
$$

C

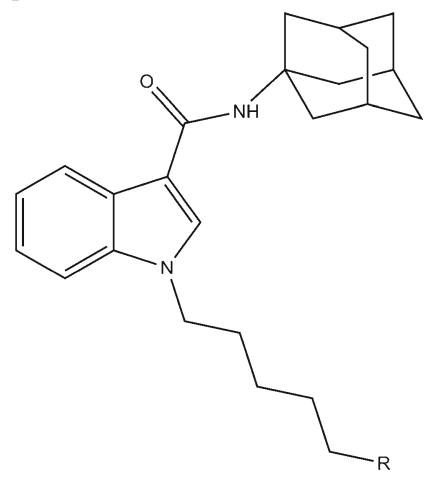

$\mathrm{R}=\mathrm{H}:$ APICA (SDB-001)

$\mathrm{R}=\mathrm{F}:$ 5F-APICA (STS-135) d

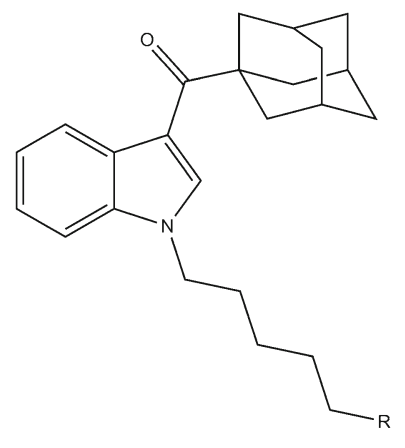

$$
\begin{aligned}
& \mathrm{R}=\mathrm{H}: \quad \mathrm{AB}-001 \\
& \mathrm{R}=\mathrm{F}: \quad 5 \mathrm{~F}-\mathrm{AB}-001
\end{aligned}
$$

Fig. 1. a-d Synthetic cannabinoid chemical structures

enzymes responsible with minimal contributions from CYP2C19, 2D6, 2E1, and 3A4 (14,15). However, as synthetic cannabinoids become increasingly structurally diverse through inclusion of new sub- and functional groups, this may potentially lend the molecules amenable to other CYP enzymes or to non-CYP-mediated biotransformations. This was recently demonstrated for the quinolineindole synthetic cannabinoids PB-22, 5F-PB-22, and BB-22, where carboxylesterase 1 (CES1) hydrolyzes an ester bond (16). In addition, CES1 also hydrolyzes the primary amide group of two other synthetic cannabinoids-AB-PINACA and ABFUBINACA (16).

This study aimed to elucidate the CYP enzymes involved in the oxidative metabolism of the synthetic cannabinoid $N$ (1-adamantyl)-1-pentyl-1 $H$-indazole-3-carboxamide (AKB48, Fig. 1b), also known as APINACA. AKB-48 deviates from earlier JWH-type synthetic cannabinoids by having an adamantyl group connected to an indazole moiety through a carboxamide linkage. The adamantyl group is also present in other synthetic cannabinoids, e.g., APICA (SDB-001, Fig. 1c), 5F-APICA (STS-135, Fig. 1c), and AB-001 (Fig. 1d). Metabolites of AKB-48 (17), 5F-AKB-48 (18), APICA (19), 5FAPICA $(19,20)$, and AB-001 (21) have been reported, but so far not the enzymes responsible for their generation.

AKB-48 was first identified in herbal blends in 2012 (4). In the USA, the Drug Enforcement Administration (DEA) temporarily listed AKB-48 as a Schedule I drug as of May 2013 (17), and the compound is also prohibited in Denmark (22). During the first half of 2013, AKB-48 and its fluorinated derivative, 5F-AKB-48 (Fig. 1b), constituted 1.0 and $2.5 \%$, respectively, of all synthetic cannabinoids reported through the DEA-operated National Forensic Laboratory Information System (NFLIS) in the USA (23). In the same year, the two compounds were identified in a total of nine drug seizures in Denmark ( $43 \%$ of all cases positive for a synthetic cannabinoid) compared to none the previous year (24). This could suggest country-specific differences in consumption patterns, which could, however, also rapidly change, e.g., due to altered consumer demand. AKB-48 and 5F-AKB-48 continue to be seized in the EU as of 2014 (EU Early Warning system, unpublished information).

\section{MATERIALS AND METHODS}

\section{Chemicals and Reagents}

Methanol and water (LC-MS grade) were from Fisher Scientific (Leicestershire, UK). Ammonium formate and formic acid were from Sigma-Aldrich Chemie (Steinheim, Germany). Reference standards of AKB-48 and JWH- $018 \mathrm{~N}$-COOH-d4 were synthesized by Chiron (Trondheim, Norway). AKB-48 N(4-hydroxypentyl) and AKB-48 N-(5-hydroxypentyl) were from Cayman Chemical (Ann Arbor, MI, USA). Inhibitors used were ketoconazole (Actavis, Gentofte, Denmark), quinidine (Nycomed, Roskilde, Denmark), and ticlopidine (Sigma-Aldrich, St. Louis, MO, USA). CYP-specific probe substrates were phenacetin (Dr. Ehrenstorfer, Augsburg, Germany), bupropion (GlaxoSmithKline, Brøndby, Denmark), dextromethorphan (Lipomed, Arlesheim, Germany), diclofenac (Afotex, Weston, Ontario), omeprazole (Astra Hässle, Mölndal, Sweden), and midazolam and chlorzoxazone (both from Cerilliant, Round Rock, TX, USA). All compounds were dissolved in methanol and stored at $-20^{\circ} \mathrm{C}$ except ticlopidine, which was dissolved in water and stored at $4^{\circ} \mathrm{C}$ for no longer than $24 \mathrm{~h}$. Phosphate buffer $(0.5 \mathrm{M})$ was prepared by dissolving $8.85 \mathrm{~g} \mathrm{~K}_{2} \mathrm{HPO}_{4} \cdot 3 \mathrm{H}_{2} \mathrm{O}$ and $1.52 \mathrm{~g} \mathrm{KH}_{2} \mathrm{PO}_{4}$ in $100 \mathrm{~mL}$ water and adjusting $\mathrm{pH}$ to 7.4 with $\mathrm{H}_{3} \mathrm{PO}_{4}$. UltraPool ${ }^{\mathrm{TM}}$ human liver microsomes (HLM) from 150 donors $(20 \mathrm{mg} / \mathrm{mL})$, CYP Supersomes ${ }^{\mathrm{TM}}$ recombinant human cDNA-expressed CYP enzymes from baculovirusinfected insect cells (CYP content 1000 or $2000 \mathrm{pmol} / \mathrm{mL}$ ), and NADPH regenerating system solutions $\mathrm{A}$ and $\mathrm{B}$ were all purchased from Corning (Woburn, MA, USA). Enzymes and NADPH regenerating system were stored at -80 and $-20^{\circ} \mathrm{C}$, respectively.

\section{Human Liver Microsomal Incubations with Inhibitors}

Incubations were conducted at $37^{\circ} \mathrm{C}$ in 96 -well twin.tec PCR plates (Eppendorf, Hamburg, Germany) sealed with flat cap strips (4titude, Surrey, UK) under shaking on a Thermomixer comfort (Eppendorf, Hamburg, Germany). The incubation mixture consisted of $1 \mathrm{mg}$ protein $/ \mathrm{mL}$, $1.3 \mathrm{mM} \mathrm{NADP}{ }^{+}, 3.3 \mathrm{mM}$ glucose-6-phosphate, $3.3 \mathrm{mM}$ 
$\mathrm{MgCl}_{2}, 0.4 \mathrm{U} / \mathrm{mL}$ glucose-6-phosphate dehydrogenase, and $0.05 \mathrm{mM}$ sodium citrate in $0.1 \mathrm{M}$ phosphate buffer $(\mathrm{pH} 7.4)$. The total incubation volume was $250 \mu \mathrm{L}$ with a final methanol content of $0.8 \% v / v$. The AKB-48 stock solution was first diluted 1:5 in water, and aliquots of the working solution immediately hereafter added to the HLM incubations to give a final concentration of $5 \mu \mathrm{M}$. The reversible chemical inhibitors ticlopidine, quinidine, and ketoconazole were used as recommended in the Food and Drug Administration (FDA) guidelines (25) to inhibit CYP2C19 $(50 \mu \mathrm{M})$ (26), $2 \mathrm{D} 6(5 \mu \mathrm{M})(15,27)$, and $3 \mathrm{~A} 4$ (1 and $5 \mu \mathrm{M})$ $(15,27,28)$, respectively. The compounds and inhibitors were pre-incubated with HLM for $5 \mathrm{~min}$ before addition of NADPH, which signified the start of the reaction. Incubations without NADPH served as negative controls for CYP activity. At $0,15,45$, and $60 \mathrm{~min}, 20 \mu \mathrm{L}$ aliquots of the incubations were quenched into $30 \mu \mathrm{L}$ ice-cold methanol containing JWH-018 N-COOH-d4 (100 ng/mL) as internal standard (IS). The quench PCR plate was subsequently heat-sealed, centrifuged for $10 \mathrm{~min}$ at $2000 \mathrm{~g}$ at $4^{\circ} \mathrm{C}$, and analyzed immediately hereafter by HPLC-HRMS.

\section{Recombinant CYP Enzyme Incubations}

Recombinant CYP enzyme (50 pmol/mL) activities were assayed under conditions similar to those of HLM (see above), except that the final methanol content was $2 \% v / v$. A FDA-recommended biotransformation of a specific probe substrate $(5 \mu \mathrm{M})$ was monitored along with each experiment as a positive control (25). Specifically, the biotransformations were phenacetin $O$-deethylation (CYP1A2), bupropion hydroxylation (CYP2B6), diclofenac 4'-hydroxylation (CYP2C9), omeprazole 5-hydroxylation (CYP2C19), dextromethorphan $O$-demethylation (CYP2D6), chlorzoxazone 6-hydroxylation (CYP2E1), and midazolam 1-hydroxylation (CYP3A4). Untransfected insect microsomes (mock) served as negative control for CYP activity and were used at $0.25 \mathrm{mg} / \mathrm{mL}$. For CYP2C18, no FDA-recommended probe substrate exists, while for CYP2C8, we did not have an available probe substrate in our lab.

\section{Precipitation in Phosphate Buffer}

AKB-48 $(5 \mu \mathrm{M})$ was incubated in pure $0.1 \mathrm{M}$ phosphate buffer $(0.8 \% \quad v / v$ methanol $)$ in borosilicate glass tubes (Corning, NY, USA) in a total volume of $1000 \mu \mathrm{L}$. At time points 0 and $120 \mathrm{~min}, 20 \mu \mathrm{L}$ of the AKB-48 solution was transferred to $30 \mu \mathrm{L}$ quench solution as previously described. After the last quench, $1440 \mu \mathrm{L}$ quench solution was then added into the remaining $960 \mu \mathrm{L} \mathrm{AKB}-48$ solution in the tube to give an equal ratio.

\section{HPLC-HR-MS and Data Treatment}

The LC-MS system consisted of a Dionex Ultimate 3000 UHPLC system (Thermo Scientific, Germering, Germany) and a high-resolution (HR) Q-Exactive mass spectrometer (Thermo Scientific, Bremen, Germany). For the metabolite experiments, chromatographic separation was performed on a
Hypersil Gold PFP $3-\mu \mathrm{m}(150 \times 2.1 \mathrm{~mm})$ column (Thermo Scientific, CA, USA) maintained at $40^{\circ} \mathrm{C}$. The mobile phase was composed of solvents A (water containing $10 \mathrm{mM}$ ammonium formate and $0.1 \% \mathrm{v} / v$ formic acid, adjusted to $\mathrm{pH}=3.0$ with formic acid) and $\mathrm{B}$ (methanol and $0.1 \% \mathrm{v} / \mathrm{v}$ formic acid). The gradient had a total run time of $14.5 \mathrm{~min}$ starting at $60 \% \mathrm{~B}$ for $0.5 \mathrm{~min}$ increasing to $85 \% \mathrm{~B}$ over $10 \mathrm{~min}$ and isocratic for $1 \mathrm{~min}$ before re-equilibration for $3 \mathrm{~min}$, while for the probe substrates, the starting \% B was lower. The flow rate was $0.3 \mathrm{~mL} / \mathrm{min}$, and the injection volume was $5 \mu \mathrm{L}$. For the HLM inhibitor experiments, a Kinetex Phenyl-Hexyl 2.6- $\mu \mathrm{m}(50 \times 2.1 \mathrm{~mm})$ column (Phenomenex, Torrance, CA, USA) was used. All analytes except chlorzoxazone were analyzed in positive electrospray ionization mode. Data recording and analysis were performed essentially as previously described (18) using TraceFinder 3.1 (Thermo Scientific, Waltham, MA, USA). Chemical structures and $\log \mathrm{P}$ value were drawn and calculated with ChemBioDraw (PerkinElmer, Waltham, MA, USA). Figures were made with FreeStyle 1.0 (Thermo Scientific, Waltham, MA, USA) and GraphPad Prism 6 (GraphPad Software, La Jolla, CA, USA).

\section{RESULTS}

\section{Metabolite Identification}

AKB-48 metabolites were previously characterized using human cryopreserved hepatocytes, and the major phase I metabolic pathway determined to be mono-, di-, and trihydroxylation on the adamantyl moiety alone or in combination with hydroxylation on the remaining $N$-pentylindazole moiety (17). AKB-48 has a dominating fragment at $\mathrm{m} / \mathrm{z}, 135.1168$ in the $\mathrm{MS}^{2}$ spectrum corresponding to the unmodified adamantyl cation $\left[\mathrm{C}_{10} \mathrm{H}_{15}\right]^{+}$, and the same is the case for hydroxylations on the pentylindazole part. Monohydroxylation on the adamantyl moiety is evident by fragment ions at $\mathrm{m} / \mathrm{z}$ 151.1117 and 133.1012 corresponding to a hydroxylated adamantyl cation $\left[\mathrm{C}_{10} \mathrm{H}_{15} \mathrm{O}\right]^{+}$and water loss hereof, respectively, while dihydroxylation on the adamantyl moiety is evident by a pattern of fragment ions at $\mathrm{m} / \mathrm{z}$ 167.1067, 149.0961, and 131.0855 corresponding to a dihydroxylated adamantyl cation $\left[\mathrm{C}_{10} \mathrm{H}_{15} \mathrm{O}_{2}\right]^{+}$, and two times water loss hereof, respectively $(17,18)$. Hydroxylation on the adamantyl ring can generate isomeric metabolites, which cannot be discriminated based on $\mathrm{MS}^{2}$ scans.

In addition to hydroxylation, we observed oxidation of the $N$-pentyl side chain to form a ketone and $N$-dealkylation. Only trace amounts of $N$-dealkylated metabolites were observed. Ketone formation appeared to be a minor metabolic pathway in agreement with the study by Gandhi et al. (17). Amide hydrolysis did not occur. A list of all the targeted metabolites is provided in supplementary I. To reduce the risk of overlooking a major metabolite, we additionally performed background subtraction and recorded data using alternating scans with and without collision energy, which were then searched for common fragments, but no additional metabolites were identified. Figure 2a shows the extracted ion chromatograms (EIC) for the hydroxy metabolites of AKB48 formed in HLM at 45 min. The AKB-48 parent compound eluted at $9.1 \mathrm{~min}$. 

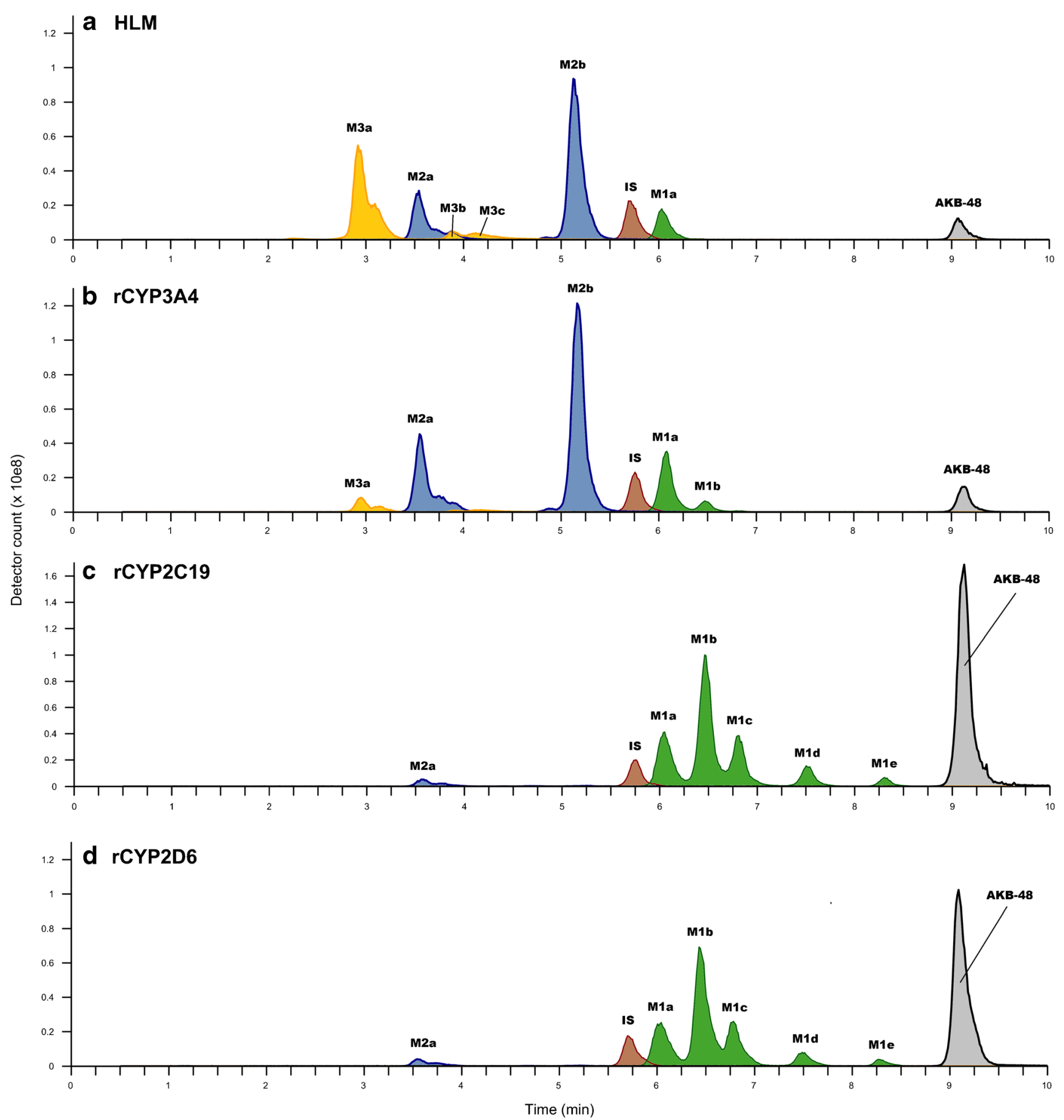

Fig. 2. EICs of AKB-48 hydroxy metabolites in HLM and recombinant CYP incubations. AKB-48 was incubated with a HLM, b rCYP3A4, c rCYP2C19, and d rCYP2D6. Aliquots of the incubations were quenched and analyzed by LC-HR-MS. Representative EICs of AKB-48 (gray), AKB-48 monohydroxy (M1: green), dihydroxy (M2: blue), and trihydroxy (M3: yellow) metabolites are shown. The peaks are labelled according to Table I. The IS EIC is shown in red. The time of incubation was 45 min for HLM and 90 min for the recombinant enzymes

\section{Screen with Recombinant CYP Enzymes}

\section{Untransfected Insect Cells (Mock)}

Incubation with microsomes from untransfected insect cells resulted in a time-dependent loss of AKB-48 signal. However, there was no evidence of formation of any metabolites, suggesting that the depletion was not caused by the compound being metabolized. AKB-48 is a hydrophobic molecule (calculated $\log P$ value of 4.48 ), and we speculated that the observed loss of signal could be due to precipitation and/or adsorption in the absence of sufficient hydrophobic membrane material.

To test this, we first incubated AKB-48 $(5 \mu \mathrm{M})$ in pure $0.1 \mathrm{M}$ phosphate buffer and quenched aliquots of the solution into the quench plate, which resulted in a gradual loss of 
signal. However, when the remaining solution was quenched in the original container to give the same ratio of quench solution (see "MATERIALS AND METHODS"), the concentration of AKB-48 was comparable to that at the beginning of the incubation, showing that no AKB-48 had been metabolized.

Increasing the total microsomal protein content from 0.25 to $1 \mathrm{mg} / \mathrm{mL}$ reduced the loss of signal. In contrast, HLM incubations of AKB-48 were stable for $60 \mathrm{~min}$ in the absence of NADPH (Fig. 3). Visual inspection of the pellet size after centrifugation revealed far more membrane material in the HLM incubations compared to the insect microsomes. This issue precluded the use of substrate depletion to assay CYP activity, and we therefore examined metabolite formation; the resulting hydroxy metabolites of AKB-48 are presented in Table I.

We used the absolute EIC peak area for each metabolite due to lack of reference standards. This approach can reasonably be expected to allow for a relative comparison of the amount of metabolite formed by different CYP enzymes. It does not, however, allow for a relative comparison of the amount of different metabolites as the ionization efficiencies may vary; assuming that the ionization efficiencies are not vastly different, this may provide an indication of major and minor metabolites.

\section{CYP1A2, 2B6, 2C8, 2C9, 2C18, and 2E1}

CYP1A2 showed limited ability to metabolize AKB-48 and formed only small amounts of monohydroxylated species (Table I); the enzyme preferentially performed the hydroxylations away from the adamantyl moiety. AKB-48 N-(5hydroxypentyl) and AKB-48 N-(4-hydroxypentyl) reference standards both eluted at $6.48 \mathrm{~min}$, so it was not possible to discriminate these two isomers (M1b). The later-eluting AKB-48 hydroxy metabolites M1c, M1d, and M1e evident at $6.84,7.48$, and $8.28 \mathrm{~min}$, respectively (Table I), are suggested to be hydroxylated on various positions on the indazole ring, since they contain the unmodified adamantyl fragment ion at $\mathrm{m} / z$ 135.1168. CYP2B6 and $2 \mathrm{C} 18 \mathrm{had}$ negligible activity towards AKB-48, while CYP2E1 showed no activity. CYP2C8 had low activity and hydroxylated both the $N$-alkyl site chain and the adamantyl moiety (M1a). CYP2C9 likewise produced small amounts of monohydroxylated metabolites with a preference for the adamantyl ring.

\section{CYP2C19, 2D6, and $3 A 4$}

CYP2C19 and 2D6 exhibited substantial activity performing monohydroxylation on both the $\mathrm{N}$-pentyl, indazole, and adamantyl moieties with no clear preference (Fig. 2c, d). The two enzymes also formed a small amount of dihydroxylated species (M2a) having one of the hydroxyl groups on the adamantyl moiety. Only trace amounts existed of the metabolite with two hydroxyl groups on the adamantyl moiety (M2b). Overall, the two enzymes displayed almost identical metabolic profiles.

CYP3A4 had the highest activity of the recombinant enzymes tested and was also the sole enzyme capable of forming trihydroxylated species (Fig. 2b); the preferred site of oxidation was the adamantyl ring as evident by the predominant formation of mono (M1a)- and dihydroxylated (M2b) adamantyl metabolites, but the enzyme also hydroxylated the $N$-pentyl side chain (M1b). In contrast, the indazole ring was not hydroxylated to a significant extent.

\section{Comparison with HLM Incubation}

The CYP phenotyping qualitatively pointed to CYP2C19, 2D6, and 3A4 as the most active enzymes in metabolizing AKB-48. Next, the formed metabolites were compared to those in HLM incubations. HLM are vesicle-like artifacts formed by remnants of the endoplasmic reticulum upon cell homogenization and have the respective enzymes in natural abundances, which are needed to correct for the relative contribution of each enzyme to the total metabolism. We used HLM from a 150-donor pool to represent an "average" population activity.

AKB-48 was completely metabolized within approximately $45 \mathrm{~min}$ in the presence of HLM and NADPH, while

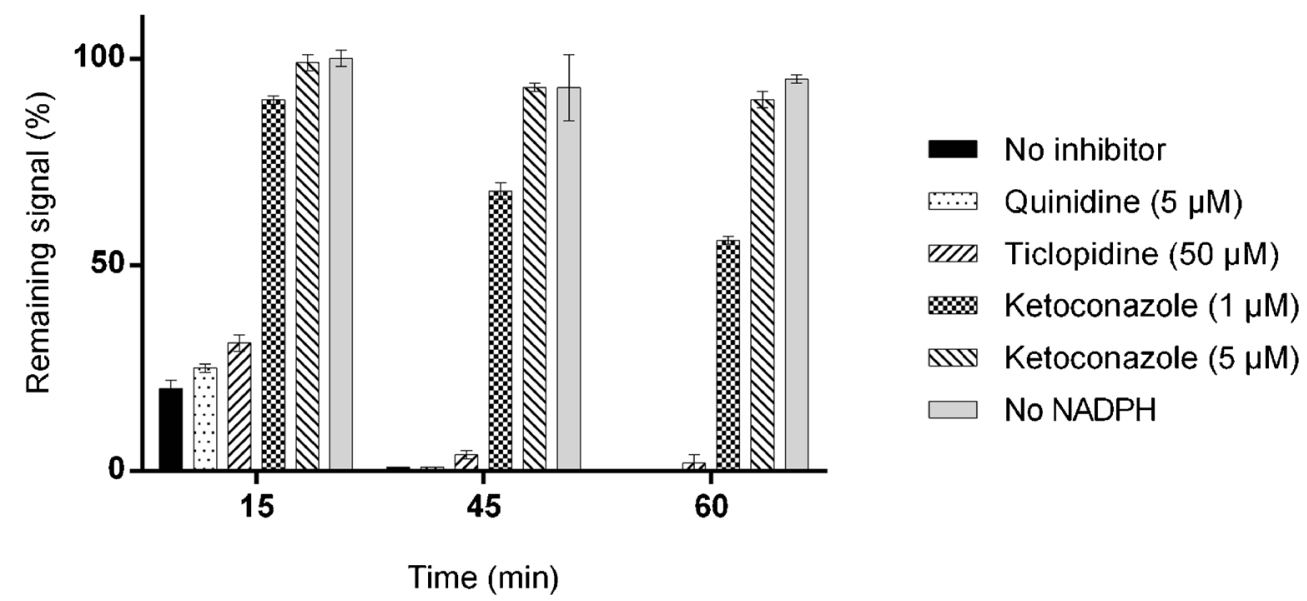

Fig. 3. AKB-48 metabolized in HLM in the presence of inhibitors. HLM were incubated with AKB-48 under the conditions specified in the legend. Aliquots of the incubations were quenched at 0, 15, 45, and $60 \mathrm{~min}$, respectively, and analyzed by LC-HR-MS. The AKB-48 signal is expressed as a percentage of the starting signal. The experiments were conducted in triplicate. Mean values are plotted with error bars representing one sample standard deviation 


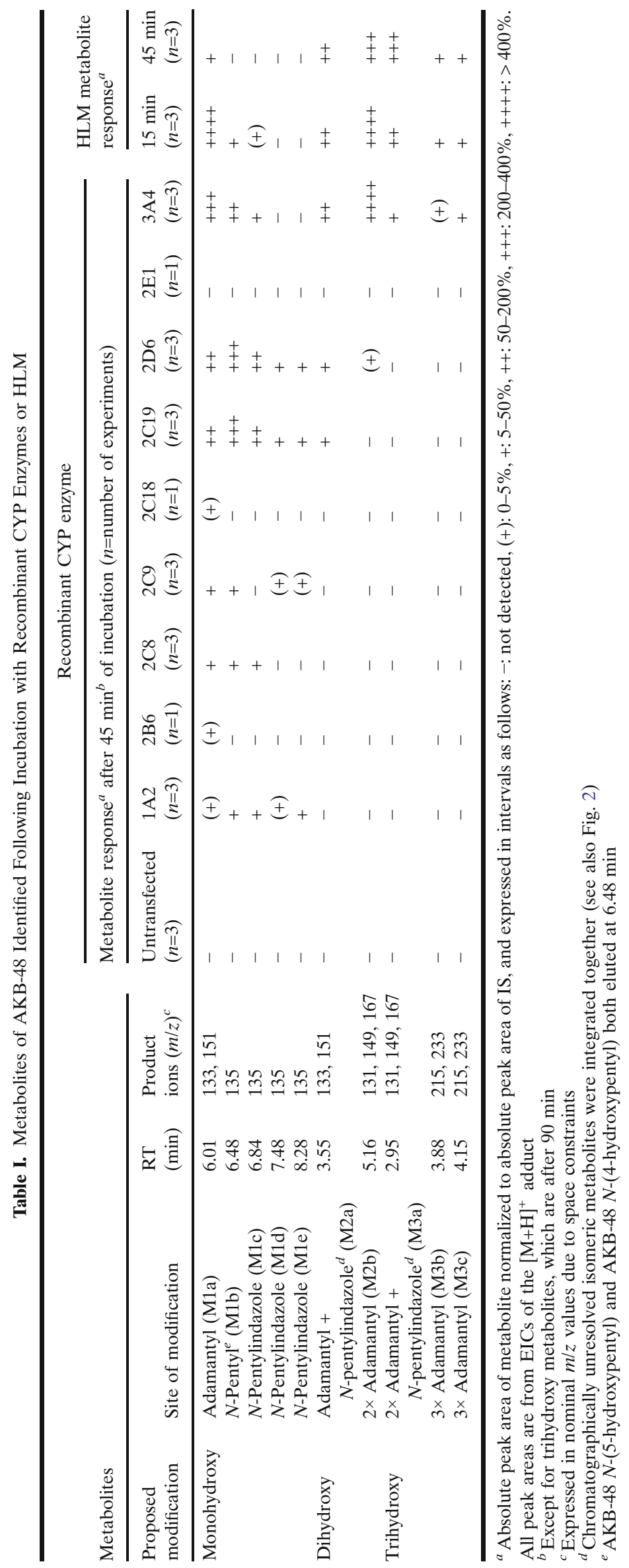


the compound was stable in the absence of NADPH for the duration of the experiment, demonstrating the involvement of CYP enzymes (Fig. 3). After 15 min of HLM incubation, the most prominent metabolites were mono- and dihydroxylated metabolites with the hydroxylations on the adamantyl moiety, while it was evident after 45 min that these metabolites were further metabolized to di- and trihydroxy metabolites, the latter of which appear to be metabolic end products (Fig. 2a). The major trihydroxylated metabolites (M3a) had two hydroxyl groups on the adamantyl ring, while three hydroxyl groups on the adamantyl ring (M3b and M3c) were less frequent. Unfortunately, we did not have full chromatographic separation of M3a, which is a limitation in this study.

To further delineate the contributions from CYP2C19, 2D6, and 3A4 in the metabolism of AKB-48, reversible chemical inhibitors were co-incubated with the HLM (Fig. 3). We used ketoconazole (CYP3A4), ticlopidine (CYP2C19), and quinidine (CYP2D6) as recommended by the FDA guidelines (25) in concentrations based on published literature values (see "MATERIALS AND METHODS"). Inclusion of quinidine $(5 \mu \mathrm{M})$ had no influence on the metabolism of AKB-48 compared to control, indicating that CYP2D6 does not participate. Ticlopidine $(50 \mu \mathrm{M})$ slowed the metabolism slightly suggesting a minor contribution from CYP2C19. The major effect, however, was obtained by inhibiting CYP3A4 with ketoconazole (Fig. 3). In the presence of $1 \mu \mathrm{M}$ ketoconazole, $56 \%$ of the starting compound remained after $60 \mathrm{~min}$, while in the absence of ketoconazole, AKB-48 was completely metabolized already after $45 \mathrm{~min}$. We could still observe mono-, di-, and trihydroxylated metabolites, suggesting that CYP3A4 was not completely inhibited at this ketoconazole concentration. We tested the ability of recombinant CYP3A4 to carry out metabolism in the presence of $1 \mu \mathrm{M}$ ketoconazole and found substantial residual activity (data not shown). The remaining metabolism in HLM could be attenuated by increasing the concentration of ketoconazole to $5 \mu \mathrm{M}$.

\section{DISCUSSION}

Screening nine recombinant CYP enzymes (CYP1A2, 2B6, 2C8, 2C9, 2C18, 2C19, 2D6, 2E1, and 3A4) for their ability to metabolize the synthetic cannabinoid AKB-48 identified CYP3A4 as the most active enzyme (Table I). CYP3A4 predominantly carried out mono- and dihydroxylation on the adamantyl ring and, to a lesser extent, on the $N$-alkyl side chain (Fig. 2b). CYP3A4 was the only enzyme forming dihydroxy metabolites to any significant degree and the sole enzyme tested capable of generating trihydroxy metabolites. CYP2C19 and 2D6, which also showed some activity, preferentially carried out hydroxylations on the $N$-pentylindazole moiety (Fig. 2c, d). The metabolites formed in HLM closely matched the metabolites formed by recombinant CYP3A4, except that the HLM generated more of the trihydroxylated species. The average microsomal CYP3A4 content has been estimated to be 60 $146 \mathrm{pmol} / \mathrm{mg}$ microsomal protein (29), and as we have used $1 \mathrm{mg} / \mathrm{mL}$ HLM, this gives a higher CYP3A4 content compared to the $50 \mathrm{pmol} / \mathrm{mL}$ in the recombinant incubations. The natural source of HLM may also result in increased enzymatic activity.
Inhibition of CYP3A4 by co-incubation with $1 \mu \mathrm{M}$ ketoconazole reduced the metabolism of AKB-48 in HLM, so that $56 \%$ of the original signal remained after 60 min (Fig. 3). In comparison, the compound was completely metabolized without the inhibitor during this time period. The remaining metabolism in HLM could be attenuated by increasing the concentration of ketoconazole to $5 \mu \mathrm{M}$ to obtain increased inhibition of CYP3A4. Co-incubation with ticlopidine had a minor inhibitory effect. Ticlopidine is a potent inhibitor of CYP2C19 and 2D6, but with marginal effect on CYP3A4 (26), and the effect could be due to simultaneous inhibition of both enzymes, which is consistent with the result from the recombinant CYP screen. However, given that inhibition of CYP2D6 with quinidine by itself showed little effect, the effect of ticlopidine may be due predominantly to inhibition of CYP2C19 at least at this substrate concentration.

Chimalakonda et al. investigated the metabolism of the JWH-type synthetic cannabinoids JWH-018 and AM-2201 using a combination of HLM and recombinant CYP enzymes. The authors concluded that CYP1A2 and 2C9 are the major hepatic CYP enzymes involved in the oxidative metabolism with minimal contribution from CYP2C19, 2D6, 2E1, and $3 \mathrm{~A} 4$. We did not find CYP1A2 or 2C9 to play a significant role in the metabolism of AKB-48, but both enzymes were capable of generating small amounts of monohydroxylated metabolites (Table I). Our results point to a major role for hepatic CYP3A4 in the metabolism of AKB-48. The adamantyl group is the preferred site of oxidation, and the presence of this moiety could potentially explain the observed differences by introducing a site of attack for CYP3A4. The active site in CYP3A4 is large and flexible and accommodates preferentially lipophilic compounds (29).

We previously characterized urinary metabolites of $5 \mathrm{~F}$ AKB-48 in an intoxication case, and most of the major metabolites were hydroxylated on the adamantyl moiety (18). Sobolevsky et al. recently reported on analysis of forensic urine samples positive for APICA (SDB-001) and 5F-APICA (STS-135), two synthetic cannabinoids differing from AKB-48 and 5F-AKB-48, respectively, by having an indole instead of an indazole ring (Fig. 1c), and the authors also identified hydroxylations on the adamantyl ring as major biotransformations (19). When assessed, recombinant CYP3A4 metabolized these three compounds in vitro and, similarly to AKB48, preferentially carried out hydroxylations on the adamantyl moiety (data not shown). These results suggest that CYP3A4 plays a key in vivo role in the metabolism of a number of adamantyl-containing synthetic cannabinoids.

A few therapeutic drugs contain adamantyl-substituted moieties. These include saxagliptin and vildagliptin, two dipeptidyl peptidase-4 (DPP-4) inhibitors used as hypoglycemic agents in the treatment of type 2 diabetes mellitus (30). The primary pathway for biotransformation of saxagliptin in humans involves CYP3A4/5-dependent monohydroxylation on the 3-hydroxy-substituted adamantyl moiety to form a 5hydroxy saxagliptin metabolite (31). The metabolism of vildagliptin, on the other hand, is virtually CYPindependent and predominantly involves hydrolysis at other parts of the molecule likely attributable to the DPP-4 target enzyme itself (32), which could explain the lack of CYP involvement. 
This study suggests that the fraction of total CYPdependent metabolism of AKB-48 mediated by CYP3A4 $\left(f_{\text {m.CYP3A4 }}\right)$ is close to 1 , and genetic polymorphisms are therefore likely not important with regard to toxicity. CYP3A4 is abundantly expressed in liver constituting an estimated average $14-24 \%$ of the total microsomal CYP pool, and is the major contributing CYP enzyme in the metabolism of approximately one third of all clinically used drugs $(29,33)$. Drugs primarily metabolized by CYP3A4 are exposed to potential DDIs through either enzyme induction or inhibition. CYP3A4 induction would increase the clearance of AKB-48 and is thus not likely to cause adverse effects. However, it should be stressed that this ultimately requires determination of whether the formed AKB-48 metabolites are devoid of biological activities. The major hydroxy metabolites of JWH-018 and AM-2201 have nanomolar $\mathrm{CB}_{1}$ receptor affinities and retain biological activities (14), and the same could be the case for AKB-48. This is in contrast to $\Delta^{9}-$ THC, where all metabolites except $11-\mathrm{OH}-\Delta^{9}$-THC are inactivated by oxidative metabolism (14).

Conversely, CYP3A4 inhibition would be expected to decrease AKB-48 clearance. The major administration route for synthetic cannabinoids is by inhalation $(12,34)$, and the compounds are thus not subjected to first-pass metabolism. Even though bioavailability may be unaffected by CYP3A4 inhibition depending on the extent of extra-hepatic metabolism, the reduced clearance rate could prolong drug circulation and increase pharmacological activity. Although pharmaceuticals that are strong CYP3A4 inhibitors are increasingly replaced with safer alternatives, several of these are still in clinical use including azole antifungals, e.g., ketoconazole and itraconazole. In addition, deliberate CYP inhibition is used as a therapeutic strategy for some HIV antivirals. This is the case for the lopinavir/ritonavir protease inhibitor cocktail, in which ritonavir boosts the plasma level of the active drug (lopinavir) by acting as a CYP3A4 inhibitor (35).

The greatest risk of intoxication, however, may still come from the uncontrolled dosing associated with abuse of these illicit drugs. This is in large part caused by considerable interand intra-batch variation in the amount of synthetic cannabinoids found in "spice" products $(2,36,37)$.

\section{CONCLUSION}

We screened a panel of nine recombinant CYP enzymes (CYP1A2, 2B6, 2C8, 2C9, 2C18, 2C19, 2D6, 2E1, and 3A4) for activity towards the synthetic cannabinoid AKB-48, and identified CYP3A4 as the major enzyme responsible for the oxidative metabolism. Recombinant CYP3A4 generated metabolites that were in close agreement with those found in HLM incubations, and the adamantyl moiety was the preferred site of oxidation. Addition of ketoconazole, a potent CYP3A4 inhibitor, attenuated the metabolism in HLM in a concentration-dependent fashion, while ticlopidine and quinidine had little or no effect, respectively. Genetic polymorphisms are likely not important with regard to toxicity given the major involvement of CYP3A4. DDIs could occur in cases with co-intake of strong CYP3A4 inhibitors, e.g., azole antifungals and HIV antivirals, which would be expected to significantly decrease the rate of metabolism of AKB-48 in vivo potentially resulting in intoxication.

\section{REFERENCES}

1. Pertwee RG. Ligands that target cannabinoid receptors in the brain: from THC to anandamide and beyond. Addict Biol. 2008;13(2):147-59.

2. Auwarter V, Dresen S, Weinmann W, Muller M, Putz M, Ferreiros N. 'Spice' and other herbal blends: harmless incense or cannabinoid designer drugs? J Mass Spectrom. 2009;44(5):832-7.

3. Uchiyama N, Kikura-Hanajiri R, Kawahara N, Haishima Y, Goda Y. Identification of a cannabinoid analog as a new type of designer drug in a herbal product. Chem Pharm Bull (Tokyo). 2009;57(4):439-41.

4. Uchiyama N, Kawamura M, Kikura-Hanajiri R, Goda Y. URB754: a new class of designer drug and 12 synthetic cannabinoids detected in illegal products. Forensic Sci Int. 2013;227(1-3):2132.

5. New psychoactive substances in Europe: An update from the EU Early Warning System (March 2015). European Monitoring Centre for Drugs and Drug Addiction. 2015. http:// www.emcdda.europa.eu/publications/2015/new-psychoactive-substances. Accessed 6 May 2015.

6. Castaneto MS, Gorelick DA, Desrosiers NA, Hartman RL, Pirard S, Huestis MA. Synthetic cannabinoids: epidemiology, pharmacodynamics, and clinical implications. Drug Alcohol Depend. 2014;144:12-41.

7. Meyer MR, Peters FT. Analytical toxicology of emerging drugs of abuse-an update. Ther Drug Monit. 2012;34(6):615-21.

8. Wohlfarth A, Weinmann W. Bioanalysis of new designer drugs. Bioanalysis. 2010;2(5):965-79.

9. Castaneto MS, Scheidweiler KB, Gandhi A, Wohlfarth A, Klette KL, Martin TM, et al. Quantitative urine confirmatory testing for synthetic cannabinoids in randomly collected urine specimens. Drug Test Anal. 2014. doi:10.1002/dta.1709.

10. Hutter M, Broecker S, Kneisel S, Auwarter V. Identification of the major urinary metabolites in man of seven synthetic cannabinoids of the aminoalkylindole type present as adulterants in 'herbal mixtures' using LC-MS/MS techniques. J Mass Spectrom. 2012;47(1):54-65.

11. Chimalakonda KC, Bratton SM, Le VH, Yiew KH, Dineva A, Moran $\mathrm{CL}$, et al. Conjugation of synthetic cannabinoids JWH-018 and JWH073, metabolites by human UDP-glucuronosyltransferases. Drug Metab Dispos. 2011;39(10):1967-76.

12. Forrester MB, Kleinschmidt K, Schwarz E, Young A. Synthetic cannabinoid and marijuana exposures reported to poison centers. Hum Exp Toxicol. 2012;31(10):1006-11.

13. Holm NB, Pineda RS, Andersen DW, Rasmussen BS, Dalsgaard PW, Hoegberg L, et al. Screening of Danish traffic cases for synthetic cannabinoids in whole blood by LC-MS/MS. Scand J Forensic Sci. 2013;19(2):45-51.

14. Chimalakonda KC, Seely KA, Bratton SM, Brents LK, Moran CL, Endres GW, et al. Cytochrome P450-mediated oxidative metabolism of abused synthetic cannabinoids found in K2/spice: identification of novel cannabinoid receptor ligands. Drug Metab Dispos. 2012;40(11):2174-84.

15. Chimalakonda KC, James LP, Radominska-Pandya A, Moran JH. Sulfaphenazole and alpha-naphthoflavone attenuate the metabolism of the synthetic cannabinoids JWH-018 and AM2201 found in K2/spice. Drug Metab Lett. 2013;7(1):34-8.

16. Thomsen R, Nielsen LM, Holm NB, Rasmussen HB, Linnet K. Synthetic cannabimimetic agents metabolized by carboxylesterases. Drug Test Anal. 2014. doi:10.1002/dta.1731.

17. Gandhi AS, Zhu M, Pang S, Wohlfarth A, Scheidweiler KB, Liu $\mathrm{HF}$, et al. First characterization of AKB-48 metabolism, a novel synthetic cannabinoid, using human hepatocytes and highresolution mass spectrometry. AAPS J. 2013;15(4):1091-8.

18. Holm NB, Pedersen AJ, Dalsgaard PW, Linnet K. Metabolites of 5 F-AKB-48, a synthetic cannabinoid receptor agonist, identified in human urine and liver microsomal preparations using liquid chromatography high-resolution mass spectrometry. Drug Test Anal. 2015;7(3):199-206.

19. Sobolevsky T, Prasolov I, Rodchenkov G. Study on the phase I metabolism of novel synthetic cannabinoids, APICA and its fluorinated analogue. Drug Test Anal. 2015;7(2):131-42. 
20. Gandhi AS, Wohlfarth A, Zhu M, Pang S, Castaneto M, Scheidweiler KB, et al. High-resolution mass spectrometric metabolite profiling of a novel synthetic designer drug, N(adamantan-1-yl)-1-(5-fluoropentyl)-1H-indole-3-carboxamide (STS-135), using cryopreserved human hepatocytes and assessment of metabolic stability with human liver microsomes. Drug Test Anal. 2015;7(3):187-98.

21. Grigoryev A, Kavanagh P, Melnik A. The detection of the urinary metabolites of 3-[(adamantan-1-yl)carbonyl]-1-pentylindole (AB001), a novel cannabimimetic, by gas chromatography-mass spectrometry. Drug Test Anal. 2012;4(6):519-24.

22. Bekendtgørelse om euforiserende stoffer. BEK 557 af 31/05/ 2011. Ministeriet for Sundhed og Forebyggelse. https:// www.retsinformation.dk/Forms/R0710.aspx?id=138271\#B1. Accessed 8 Apr 2015.

23. 2.5 Synthetic cannabinoids. In: 2013 Annual Report. National Forensic Laboratory Information System. 2014. https:// www.nflis.deadiversion.usdoj.gov/DesktopModules/ ReportDownloads/Reports/NFLIS2013AR.pdf. Accessed 8 Apr 2015.

24. Illegale stoffer i Danmark. In: Illegale stoffer i Danmark, Årsrapport 2013. Sundhedsstyrelsen. 2014. https:// sundhedsstyrelsen.dk/da/sundhed/narkotika/indhold-i-illegalestoffer /media/0F7441B6213B4FA3A8E874B00984B964.ashx. Accessed 8 Apr 2015.

25. Drug Development and Drug Interactions: Table of substrates, inhibitors and inducers. In: Development \& approval process (drugs). U.S. Food and Drug Administration. 2014. http://www.fda.gov/drugs/ developmentapprovalprocess/developmentresources/ druginteractionslabeling/ucm093664.htm. Accessed 8 Apr 2015.

26. Ko JW, Desta Z, Soukhova NV, Tracy T, Flockhart DA. In vitro inhibition of the cytochrome P450 (CYP450) system by the antiplatelet drug ticlopidine: potent effect on CYP2C19 and CYP2D6. Br J Clin Pharmacol. 2000;49(4):343-51.

27. Olesen OV, Linnet K. Identification of the human cytochrome $\mathrm{P} 450$ isoforms mediating in vitro $\mathrm{N}$-dealkylation of perphenazine. Br J Clin Pharmacol. 2000;50(6):563-71.
28. Yamazaki H, Inoue K, Shaw PM, Checovich WJ, Guengerich FP, Shimada T. Different contributions of cytochrome P450 2C19 and $3 \mathrm{~A} 4$ in the oxidation of omeprazole by human liver microsomes: effects of contents of these two forms in individual human samples. J Pharmacol Exp Ther. 1997;283(2):434-42.

29. Zanger UM, Schwab M. Cytochrome P450 enzymes in drug metabolism: regulation of gene expression, enzyme activities, and impact of genetic variation. Pharmacol Ther. 2013;138(1):103-41.

30. Scheen AJ. Dipeptidylpeptidase-4 inhibitors (gliptins): focus on drug-drug interactions. Clin Pharmacol Ther. 2010;49(9):573-88.

31. Su H, Boulton DW, Barros Jr A, Wang L, Cao K, Bonacorsi Jr SJ, et al. Characterization of the in vitro and in vivo metabolism and disposition and cytochrome P450 inhibition/induction profile of saxagliptin in human. Drug Metab Dispos. 2012;40(7):1345-56.

32. He H, Tran $\mathrm{P}$, Yin $\mathrm{H}$, Smith $\mathrm{H}$, Batard Y, Wang L, et al. Absorption, metabolism, and excretion of [14C]vildagliptin, a novel dipeptidyl peptidase 4 inhibitor, in humans. Drug Metab Dispos. 2009;37(3):536-44.

33. Anzenbacher P, Anzenbacherova E. Cytochromes P450 and metabolism of xenobiotics. Cell Mol Life Sci. 2001;58(5-6):73747.

34. Forrester MB, Kleinschmidt K, Schwarz E, Young A. Synthetic cannabinoid exposures reported to Texas poison centers. J Addict Dis. 2011;30(4):351-8.

35. Zhou X, Gao ZW, Meng J, Chen XY, Zhong DF. Effects of ketoconazole and rifampicin on the pharmacokinetics of GLS4, a novel anti-hepatitis B virus compound, in dogs. Acta Pharmacol Sin. 2013;34(11):1420-6.

36. Dresen S, Ferreiros N, Putz M, Westphal F, Zimmermann R, Auwarter V. Monitoring of herbal mixtures potentially containing synthetic cannabinoids as psychoactive compounds. J Mass Spectrom. 2010;45(10):1186-94.

37. Hudson S, Ramsey J, King L, Timbers S, Maynard S, Dargan PI, et al. Use of high-resolution accurate mass spectrometry to detect reported and previously unreported cannabinomimetics in "herbal high" products. J Anal Toxicol. 2010;34(5):252-60. 